\title{
Selective suppression of the JNK-MMP2/9 signal pathway by tetramethylpyrazine attenuates neuropathic pain in rats
}

Lai Jiang ${ }^{1 \dagger}$, Cai-Long Pan ${ }^{1 \dagger}$, Chao-Yu Wang ${ }^{1}$, Bing-Qian Liu², Yuan Han ${ }^{3}$, Liang Hu' ${ }^{1}$, Lei Liư ${ }^{4}$, Yang Yang ${ }^{5}$, Jun-Wei Qu ${ }^{5}$ and Wen-Tao Liu ${ }^{1 *}$

\begin{abstract}
Background: Activated astrocytes release matrix metalloproteinase-2/9 (MMP-2/9) to induce central sensitization and maintain neuropathic pain. However, the mechanisms involved in the activation of MMP-2/9 on astrocytes during pain remain poorly understood. Meanwhile, there is a lack of effective treatment to inhibit the activation of MMP-2/9 on astrocytes. In this study, we aim to investigate the effect of tetramethylpyrazine (TMP), a natural compound with analgesic effects but unknown mechanisms, on MMP-2/9 in neuropathic pain.
\end{abstract}

Methods: The nociception was assessed by measuring the incidence of foot withdrawal in response to mechanical indentation in rats $(n=6)$. Cell signaling was assayed using western blotting $(n=6)$ and immunohistochemistry $(n=5)$. The astrocyte cell line C8-D1A was cultured to investigate the in vitro effects.

Results: TMP significantly attenuated the maintenance of chronic constrictive injury (CCI)-induced neuropathic pain, inhibited the activation of astrocytes, and decreased the expression of MMP-2/9. Furthermore, our results indicated that TMP could selectively suppress JNK activity but had no notable effects on ERK and p38. Our study also revealed that the effect of TMP may be dependent on the inhibition of TAK1.

Conclusions: Inhibition of astrocyte activation in the spinal cord by tetramethylpyrazine may have utility in the treatment of CCl-induced neuroinflammation, and our results further implicate JNK-MMP-2/9 as a novel target for the attenuation of neuropathic pain.

\section{Background}

Neuropathic pain is defined as pain caused by a lesion or disease of the somatosensory nervous system [1]. Management of neuropathic pain remains a clinical challenge: current treatments for chronic pain have been greatly limited by an incomplete understanding of its pathogenesis. The continuous search for a safer and more effective compound is urgently needed.

Convincing evidence shows that nerve injury induces the profound activation of glial cells, including microglia and astrocytes, in the spinal cord [2]. Microglia and astrocytes both play important roles in neuropathic pain.

\footnotetext{
* Correspondence: painresearch@njmu.edu.cn

${ }^{\dagger}$ Equal contributors

'Department of Pharmacology, Jiangsu Key Laboratory of

Neurodegeneration, Nanjing Medical University, 140 Han-Zhong Road,

Nanjing 210029, China

Full list of author information is available at the end of the article
}

Activation of spinal microglial cell occurs in the early phase after injury and is critical in the induction of neuropathic pain [3]. Compared with the microglial response, astrocytes are activated in the late phase after injury and are thought to be important in the maintenance of neuropathic pain [3]. Astrocyte proliferation begins relatively late and progresses slowly but is sustained for a longer period (more than 5 months) [4]. Further studies indicate that intrathecal injection of the astrocyte inhibitor fluoroacetate could significantly alleviate behaviors associated with neuropathic pain in animal models [5].

Activated astrocytes produce numerous proinflammatory cytokines (such as IL-1 $\beta$ and TNF- $\alpha$ ) [6], chemokines (such as CX3CL1 (fractalkine) and CCL2 (MCP-1)) [7], and algogenic substance (such as MMP-2/9) [8] to facilitate central sensitization [9]. Among all the pain-related substrates, MMP-2/9 has attracted increasing attention 
for neuropathic pain [10]. MMP-9 contributes to the early stage of neuropathic pain, while MMP-2 maintains neuropathic pain [11]. On the one hand, MMP-2/9 contributes to the maturation of IL- $1 \beta$, which increases NMDA receptor phosphorylation via a PKC $\gamma$-dependent manner [10]. On the other hand, a previous study by our group showed that spinal MMP-9 could enhance the activation of $N$ methyl-D-aspartate (NMDA) receptors via the integrin$\beta 1$-mediated signal cascade [12]. Hence, there is an urgent need for a safe and effective astrocyte and MMP-2/9 inhibitors that can be used in the clinic for the treatment of neuropathic pain.

Based on the information mentioned above, preventing the activation of astrocytes and MMP-2/9 is becoming an attractive target for suppressing neuropathic pain. Our group is focused on searching for an "old" drug, which, historically, has been clinically effective and safe, to manage neuropathic pain via inhibition of MMP-2/9. After screening a variety of compounds and based on our group's preliminary data, we focused on tetramethylpyrazine (TMP), the bioactive component extracted from chuanxiong (Ligusticum chuanxiong hort). The reason we choose TMP as a research target is based on the following considerations: First, chuanxiong, a Chinese traditional medicine, has been used for treating chronic pain for more than 100 years [13]. Second, TMP can significantly decrease the migration and proliferation of glioma cells in rats [14]. Additionally, it is widely known that high MMP$2 / 9$ expression is a key characteristic of gliomas, which could significantly accelerate remodeling [13], development, and angiogenesis [15]. Third, TMP has been widely used for three decades in clinical treatments, including ischemic and cerebral infarction diseases of the central nervous system to suppress neuroinflammation [14]. Therefore, we hypothesized that TMP might suppress chronic constrictive injury (CCI)-induced MMP-2/9 from astrocytes. In the current study, we examined whether TMP inhibits MMP-2/9 in astrocytes.

\section{Methods}

\section{Ethics statement}

All procedures were strictly performed in accordance with the regulations of the ethics committee of the International Association for the Study of Pain and the Guide for the Care and Use of Laboratory Animals (The Ministry of Science and Technology of China, 2006). All animal experiments were approved by Nanjing Medical University Animal Care and Use Committee and were designed to minimize the suffering and the number of animals used.

\section{Animals and neuropathic pain model}

Adult male Sprague-Dawley rats (180-200 g wt.) were provided by the Experimental Animal Center at Nanjing Medical University, Nanjing, China. Animals were housed five to six per cage under pathogen-free conditions with soft bedding under controlled temperature $\left(22 \pm 2{ }^{\circ} \mathrm{C}\right)$ and a 12-h light/dark cycle (lights on at 8:00 a.m.). Behavioral testing was performed during the light cycle (between 9:00 a.m. and 5:00 p.m.). The animals were allowed to acclimate to these conditions for at least 2 days before starting experiments. Animals were randomly divided into groups $(n=6)$. The sample size was designed on prior experience and to be limited to the minimal as scientifically justified). For each group of experiments, the animals were matched by age and body weight. All surgeries were done under anesthesia induced by chloral hydrate $(300 \mathrm{mg} / \mathrm{kg}$, i.p.). Peripheral nerve injury was imitated by the model of chronic constriction injury $(\mathrm{CCI})$ of the sciatic nerve. In brief, the left common sciatic nerve of each rat was exposed at the mid-thigh level. Proximal to the sciatic nerve's trifurcation, approximately $7 \mathrm{~mm}$ of nerve was separated from adhering tissue and four ligatures (4-0 chronic gut) were tied loosely around it with about $1 \mathrm{~mm}$ between ligatures. After surgery, the skin layers and muscle were sutured, and the surgery area was sterilized with iodine.

\section{Drugs and reagents}

Tetramethylpyrazine was purchased from Zelang Pharmaceutical Co. Ltd. (Nanjing, China). The purity of tetramethylpyrazine was more than $99 \%$. TMP was dissolved in sterile saline $(0.9 \%)$, the allocation of different concentrations of TMP solution, and the concentration of TMP was 1,3 , and $9 \mathrm{mg} / \mathrm{ml}$. Solvent-treated controls were injected with sterile saline. Fetal bovine serum (FBS) and other cell culture media and supplements were purchased from Hyclone (USA). SP600125 was purchased from beyotime biotechnology, and 5Z-7-oxozeaenol was purchased from Millipore. Anti-glyceraldehyde 3phosphatedehydrogenase (GAPDH) was from Sigma. Anti-Phospho-p38 MAPK was from Cell Signaling Technology (Beverly, MA, USA). Anti-ionized calciumbinding adapter molecule 1 (IBA-1), anti-JNK, antiMMP9, and anti-MMP2 were from Abcam (USA). Anti-phosphorylated $N$-methyl-D-aspartate receptor (NMDAR) NR1 subunit (Ser896) was from Cell Signaling Technology (Beverly, MA, USA). Anti-phospho-PKC (pan) (gamma Thr514) Antibody was from Cell Signaling Technology (Beverly, MA, USA). Anti-GFAP, anti-p44/ 42 MAPK (Erk 1/2), and anti-p38 MAPK were from Cell Signaling Technology (Beverly, MA, USA). AntiPhospho-p44/42 MAPK (Erk1/2) was from Cell Signaling Technology (Beverly, MA, USA). Anti-phosphoSAPK/ JNK was from Cell Signaling Technology (Beverly, MA, USA). TAK1 was from Cell Signaling Technology (Beverly, MA, USA). Secondary antibodies were from Cell Signaling Technology (Beverly, MA, USA). All other chemicals were purchased from Sigma. 


\section{Cell culture}

Astrocyte C8-D1A cells were incubated under humidified $5 \% \mathrm{CO} 2$ and $95 \% \mathrm{O} 2$ at $37{ }^{\circ} \mathrm{C}$ in Dulbecco's modified Eagle's medium (DMEM; Invitrogen, USA) containing 10\% FBS and 1\% streptomycin and penicillin (Invitrogen). Twenty-four hours before experimentation, the culture media was replaced by $0.5 \%$ FBS highglucose DMEM. Then, the cells were stimulated with IL-1 $\beta(20 \mathrm{ng} / \mathrm{ml})$ for $30 \mathrm{~min}$ with or without tetramethylpyrazine $(0.5,5$, and $50 \mu \mathrm{M})$.

\section{Intrathecal injection procedure}

To perform intrathecal (i.t.) injections, the rat was placed in a prone position and the midpoint between the tips of the iliac crest was located. A Hamilton syringe with 30-gauge needle was inserted into the subarachnoid space of the spinal cord between the L5 and L6 spinous processes. Proper intrathecal injection was systemically confirmed by observation of a tail flick. Intrathecal injection did not affect baseline responses, compared with latencies recorded before injection.

\section{Gelatin zymography}

Animals were anesthetized deeply with chloral hydrate (300 mg/kg, i.p.), and spinal cord segments (L1-L6) were rapidly dissected and homogenized in 1\% NP40 lysis. 300-500 $\mu \mathrm{g}$ of protein per lane was loaded into the wells of precast gels ( $8 \%$ polyacrylamide gels containing $0.1 \%$ gelatin). After electrophoresis, each gel was incubated with $50 \mathrm{ml}$ of developing buffer for $48 \mathrm{~h}\left(37.5{ }^{\circ} \mathrm{C}\right)$ in shaking bath. Then, the gels were stained with coomassie brilliant blue (1\%, with $10 \%$ acetic acid, $10 \%$ isopropyl alcohol, diluted with dd $\mathrm{H} 2 \mathrm{O})$.

\section{Western blotting}

The entail spinal cord segments at L1-L6 were rapidly collected at $4 \mathrm{~h}$ after the last drug administration. The protein concentrations were determined by BCA Protein Assay (Thermo Fisher, Waltham, MA, USA), and 40$80 \mu \mathrm{g}$ of proteins were loaded and separated by SDSPAGE and electrophoretically transferred onto polyvinylidene fluoride membranes (Millipore Corp., Bedford, MA). The membranes were blocked with $5 \%$ bovine serum albumin for $1 \mathrm{~h}$ at room temperature, probed with antibodies overnight at $4{ }^{\circ} \mathrm{C}$ with the primary antibodies and then incubated with HRP-coupled secondary antibodies. The primary antibodies used included IL-1 $\beta$ (1:300), p-NR1(1:1000), p-PKCץ (1:1000), p-p38 (1:1000), p-JNK(1:1000), p-ERK (1:1000), p-TAK1(1:1000). For loading control, the blots were probed with antibody for GAPDH (1:8000). The filters were then developed by enhanced chemiluminescence reagents (Perkinelmer) with secondary antibodies (Chemicon). Data were analyzed with the Molecular Imager (Gel DocTM XR, 170-8170) and the associated software Quantity One-4.6.5(Bio-Rad Laboratories, Berkeley, CA).

\section{Behavioral analysis}

Animals were habituated to the testing environment daily for at least 2 days before baseline testing. Mechanical sensitivity was detected by von Frey Hairs (Woodland Hills, LA, USA) test. Animals were placed in boxes set on an elevated metal mesh floor and were allowed $30 \mathrm{~min}$ for habituation before testing. The plantar surface of each hind paw was stimulated with a series of von Frey hairs with logarithmically incrementing stiffness perpendicularly to the plantar surface. Each rat was tested for three times, and the average of the threshold was measured.

\section{Immunofluorescence}

After deep anesthesia by intraperitoneal injection of chloral hydrate $(300 \mathrm{mg} / \mathrm{kg})$, the animal was perfused transcardially with normal saline followed by $4 \%$ paraformaldehyde in $0.1 \mathrm{M} \mathrm{PB}, \mathrm{pH} 7.4$, each for $20 \mathrm{~min}$. Then, L4 and/or L5 lumbar segment was dissected out and post-fixed in $4 \%$ paraformaldehyde. The embedded blocks were sectioned as $25 \mu \mathrm{m}$ thick. Sections from each group (five mice in each group) were incubated with goat antibodies for GFAP (1:200), p-JNK(1:100). Then, the free-floating sections were washed with PBS and incubated with the secondary antibody for $2 \mathrm{~h}$. After washing out three times with PBS, the samples were studied under an immunofluorescence microscope (Zeiss AX10, Germany) for morphologic details of the immunofluorescence staining. Examination was blindly carried out. Images were randomly coded, and the fluorescence intensities were analyzed by Image Pro Plus 6.0 software (Media Cybernetics Inc. Rockville, MD, USA). The average green fluorescence intensity of each pixel was normalized to the background intensity in the same image.

\section{RT-PCR}

Samples (spinal cord segments at L1-L6) were homogenized in Trizol reagent (Invitrogen Life Technologies, Carlsbad, CA, USA), and total RNA was treated by DNaseI and subjected to quantitative PCR, which was performed with ABI Prism 7300 sequence detection system (Applied Biosystems, Foster City, CA, USA) using SYBR Green I dye. The sense and antisense primers used for the analysis of rat MMP-9, MMP-2, and GAPDH expression were as follows: MMP-9: 5' TCGAAGGCGACCTCAAGTG-3' and 5'-TTCGGTG TAGCTTTGGATCCA-3', MMP-2: 5' -ACCGTCGCCC ATCATCAA-3' and 5' ${ }^{\prime}$-TTGCACTGCCAACTCTTTGT 
CT-3', and GAPDH: 5'-ATGACTCTACCCACGGCAAG$3^{\prime}$ ' and $5^{\prime}$-CTGGAAGATGGT GATGGGTT-3'.

\section{Statistical analyses}

SPSS Rel 15 (SPSS Inc., Chicago, IL, USA) was used to conduct all the statistical analyses. Alteration of expression of the proteins detected and the behavioral responses were tested with one-way ANOVA, and the differences in latency over time among groups were tested with two-way ANOVA. Bonferroni post hoc tests were conducted for all ANOVA models. Results are expressed as mean \pm SD of three independent experiments. Results described as significant are based on a criterion of $P<0.05$.

\section{Results}

TMP treatment attenuated $\mathrm{CCl}$-induced neuropathic pain First, we measured the mechanical threshold of CCItreated rats using the von Frey test. Fourteen days after the CCI surgery, the mechanical threshold was markedly decreased in CCI-treated rats. A single dosage of TMP (10, 30, and $90 \mathrm{mg} / \mathrm{kg}$, i.p.) was given intraperitoneally to $\mathrm{CCI}$ rats, and the mechanical withdrawal threshold was greatly increased (Fig. 1a).

Furthermore, the action of TMP was studied by continuous administration for 5 days from the 14 days after the CCI surgery. In the CCI group, the rats' mechanical threshold was reduced. After continuous administration of TMP $(10,30$, and $90 \mathrm{mg} / \mathrm{kg}$, i.p.) for 5 days, the mechanical threshold was elevated (Fig. 1c).

To provide additional evidence, TMP was administered intrathecally to the CCI-treated rats. The mechanical allodynia was notably ameliorated by a single dose of TMP (Fig. 1b). These data partially address that a local administration targeting spinal cord mechanisms would induce similar effects to those observed after systemic administration.

\section{TMP could significantly inhibit $\mathrm{CCl}$-induced astrocyte} activation

Glial cell activation and the crosstalk between glia and neurons enhance central sensitization. We evaluated whether TMP affects the activation of glia induced by the CCI operation. Our results showed that TMP (30 $\mathrm{mg} / \mathrm{kg}$, i.p.) could significantly suppress the upregulated astrocyte marker GFAP in the spinal cord after the CCI operation (Fig. 2a). However, TMP did not show a notable influence on the microglia marker IBA1 (Additional file 1: Figure S2). Further, immunofluorescence of GFAP in the dorsal horn of CCI-treated rats showed the activation of astrocytes. Notably, this activation was alleviated by TMP (Fig. 2 b, c).

\section{Single and continuous administration of TMP-suppressed CCl-induced activation of MMP-9 and MMP-2 in vivo}

To investigate the effects of TMP on MMP-9 and MMP-2 in vivo, single dosages of TMP were given intraperitoneally to CCI rats. Gelatin zymography results showed that TMP (30 mg/kg, i.p.) could inhibit the activity of MMP-9 and MMP-2 in the spinal cord (Fig. 3a). Furthermore, the action of TMP was studied by continuous administration for 5 days from 14 days after the CCI surgery. Consecutive administration of TMP (30 $\mathrm{mg} / \mathrm{kg}$, i.p.) for 5 days significantly inhibited the activity of MMP-9 and MMP-2 in the spinal cords from CCI-treated rats (Fig. 3b). The mRNA expression of the MMP-9 and MMP-2 were increased in CCI-treated rats (Fig. 3c, d). Compared with the CCI-treated rats, single and continuous administration of TMP (30 mg/kg, i.p.) significantly reduced these effects (Fig. 3c, d). Furthermore, single and continuous administration of TMP (30 mg/kg, i.p.) could significantly reduce CCI-induced protein expression of MMP-9 and MMP-2 in the spinal cord (Fig. 3e, f).

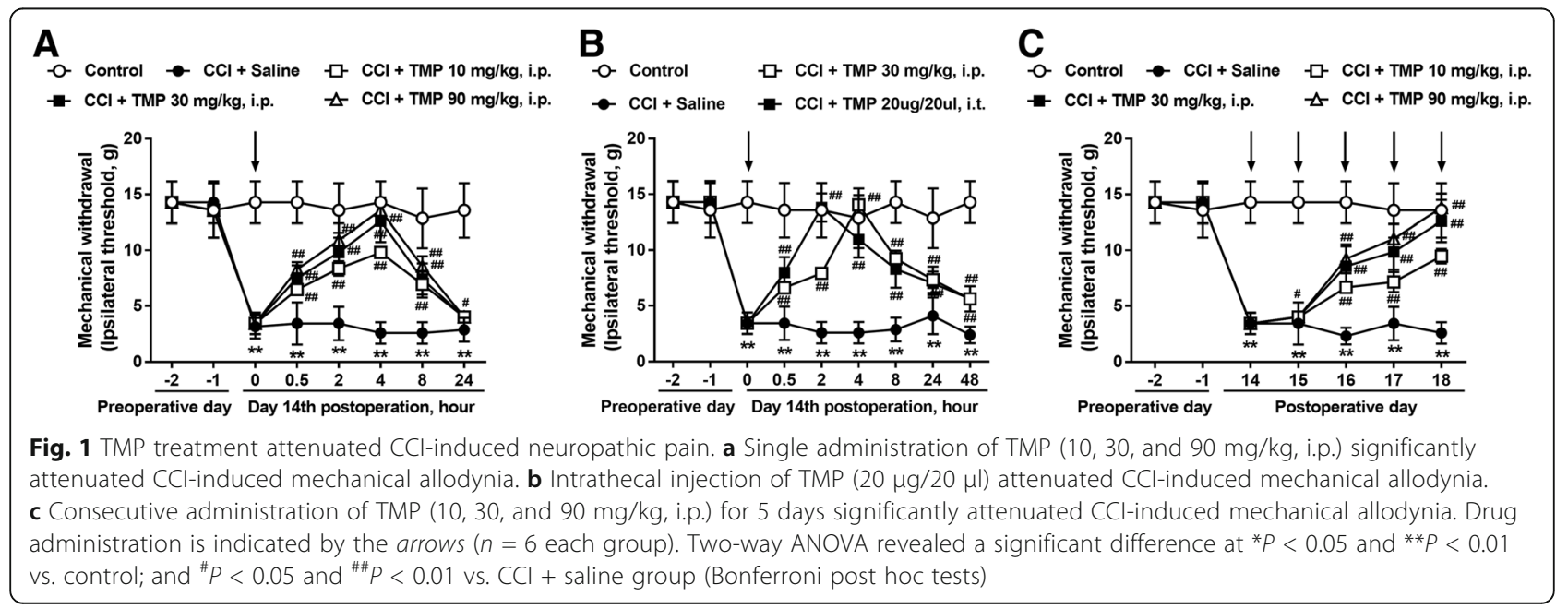



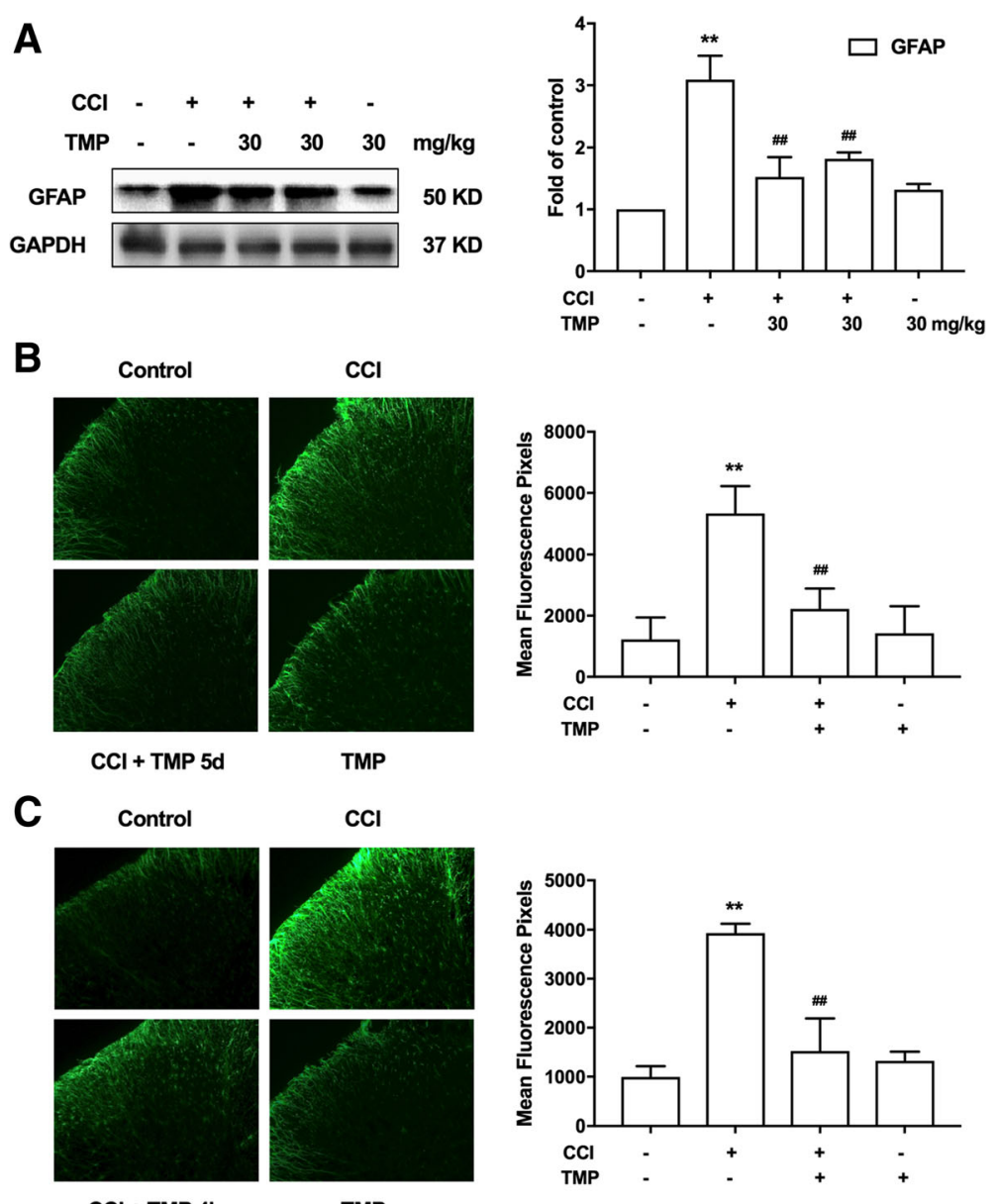

Fig. 2 TMP significantly inhibited CCl-induced activation of astrocytes. a Single administration (the third band) and consecutive administration (the fourth band) of TMP (30 mg/kg, i.p.) significantly suppressed CCl-induced expression of GFAP in the spinal cord. b Consecutive administration of TMP (30 mg/kg, i.p.) significantly suppressed CCl-induced expression of GFAP in the spinal cord. TMP (30 mg/ $\mathrm{kg}$, i.p.) was consecutively administered daily from days 14 to 18 after the CCl operation. The lumbar spines (L4-L6) were collected and analyzed at $4 \mathrm{~h}$ after the last drug administration. c Single administration of TMP (30 mg/kg, i.p.) significantly suppressed CCl-induced expression of GFAP in the spinal cord. The lumbar spines (L4-L6) were collected and analyzed at $4 \mathrm{~h}$ after TMP administration. Confocal images and immunofluorescence analysis data showing GFAP in the dorsal horns. Quantification of immunofluorescence was represented as the mean fluorescent pixels in the superficial dorsal horns. One-way ANOVA revealed a significant difference at ${ }^{*} P<0.05$ and ${ }^{* *} P<0.01$ vs. control; and ${ }^{\#} P<0.05$ and ${ }^{\# \#} P<0.01$ vs. CCl group

TMP selectively inhibited CCl-induced JNK phosphorylation in astrocytes but not p38 or ERK

Accumulating evidence shows that all three MAPK pathways contribute to pain sensitization after tissue and nerve injury via distinct molecular and cellular mechanisms. Because activation of MAPKs under different persistent pain conditions results in the induction and maintenance of pain hypersensitivity, we tested the effect of TMP on the MAPK family using western blotting. On the 14th day after the CCI operation, a single and continuous administration of TMP (30 mg/kg, i.p.) could significantly reduce CCIinduced phosphorylation of JNK in the spinal cord but not p38 or ERK (Fig. 4a, b). Immunofluorescence of GFAP and p-JNK in the dorsal horn showed a significant inhibition of TMP on CCI-induced activation of astrocytes (Fig. 4c).
TMP significantly inhibited CCl-induced IL-1 $\beta$ cleavage, PKC $Y$ phosphorylation, and NR1 phosphorylation

The above results proved that TMP significantly inhibited the activation of MMP-2/9 and attenuated chronic constrictive injury (CCI)-induced neuropathic pain. MMP-9 has the ability to cleave IL- $1 \beta$, which is essential for pain generation. Here, we reported that a single and continuous administration of TMP (30 $\mathrm{mg} / \mathrm{kg}$, i.p.) blocked the production of maturated IL-1 $\beta$ induced by CCI (Fig. $5 \mathrm{a}$ ).

IL-1 $\beta$ was expressed in DRG neurons and some satellite cells. We then evaluated the influence of TMP on neuronal activation induced by the CCI operation. TMP dramatically decreased CCI-induced phosphorylation of both PKC $\gamma$ and NR1 by single administration of TMP (30 mg/kg, i.p.) (Fig. 5b). 

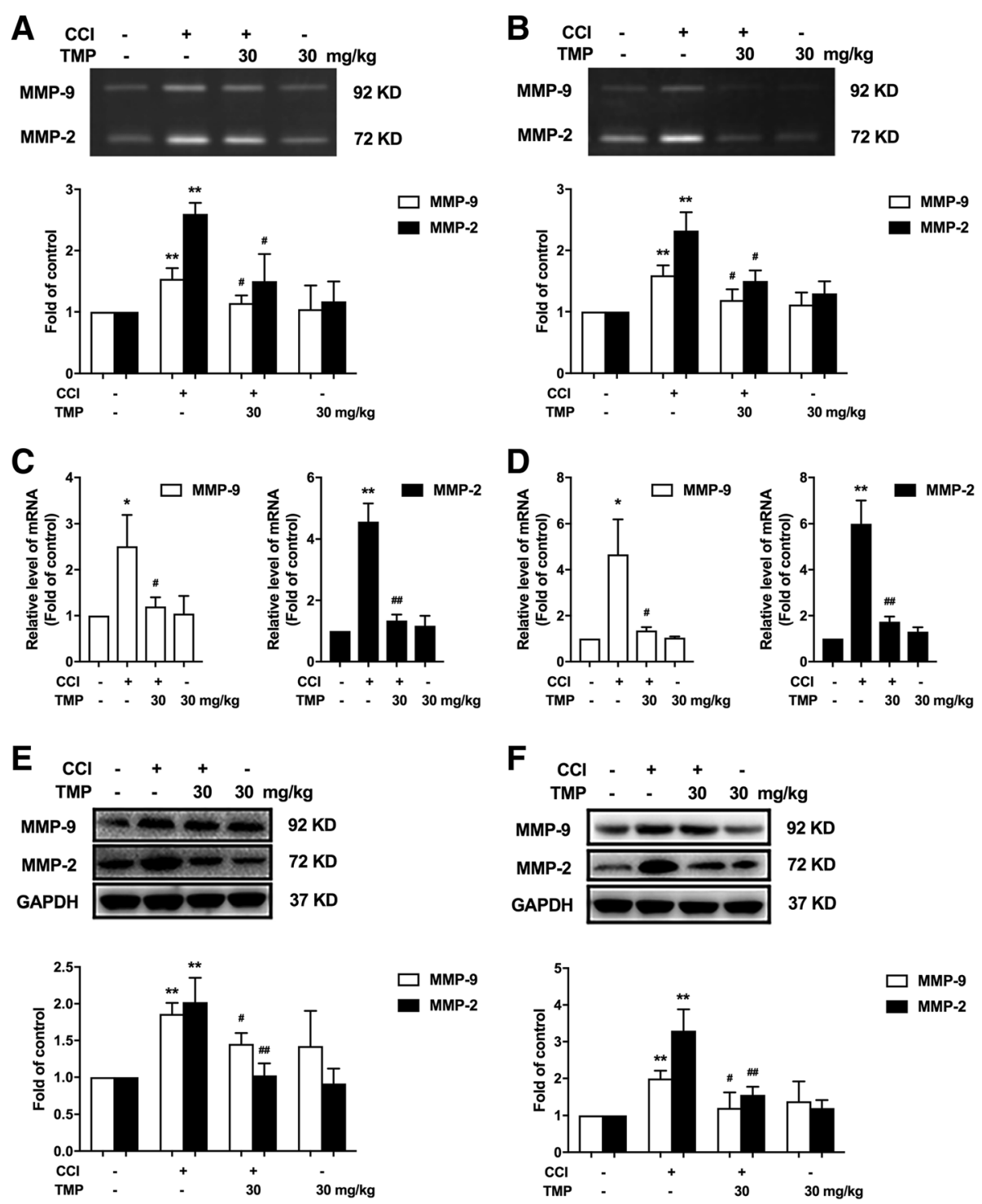

Fig. 3 Single and consecutive administration of TMP significantly suppressed CCl-induced activation of MMP-2/9 in vivo. a Single administration of TMP (30 mg/kg, i.p.) significantly suppressed CCI-induced activation of MMP-2 and MMP-9 in the spinal cords. b Consecutive administration of TMP (30 mg/kg, i.p.) for 5 days from 14 days after the CCl operation significantly attenuated CCl-induced activation of MMP-2 and MMP-9 in the spinal cord. The gelatin zymography samples $(n=6)$ were collected as described above. c Single administration of TMP (30 mg/kg, i.p.) significantly attenuated CCl-induced increase in the expression of MMP-2 and MMP-9. d Consecutive administration of TMP (30 mg/kg, i.p.) significantly attenuated CCl-induced increase in the expression of MMP-2 and MMP-9. The levels of MMP-2 and MMP-9 mRNA in the spinal cord were determined using real-time quantitative PCR. GAPDH was used as an invariant control. e Single administration of TMP (30 mg/kg, i.p.) significantly attenuated CCl-induced increase in the expression of MMP-2 and MMP-9. f Consecutive administration of TMP (30 mg/kg, i.p.) significantly attenuated CCl-induced increase in the expression of MMP-2 and MMP-9. The lumbar spines (L1-L6) were collected and analyzed using western blot. A significant difference was at ${ }^{*} P<0.05,{ }^{* *} P<0.01$ vs. control; ${ }^{\#} P<0.05,{ }^{\# \#} P<0.01$ vs. CCl group

TMP significantly inhibited CCl-induced TAK1 phosphorylation; the TAK1 and JNK inhibitors can mimic the effect of TMP

In the spinal cord, TAK1-induced activation of astrocytes is crucial for mechanical hypersensitivity after peripheral nerve injury. After nerve injury, TAK1 was increased in hyperactive astrocytes but not in neurons or microglia [16]. Here, we demonstrated that TMP (30 mg/kg, i.p.) could inhibit the activation of TAK1 phosphorylation in the spinal cords of rats in vivo (Fig. 6a). Furthermore, continuous administration of TMP (30 mg/kg, i.p.) for 5 days significantly inhibited the activity of TAK1 phosphorylation in the spinal cords of CCI-treated rats in vivo (Fig. 6a). 


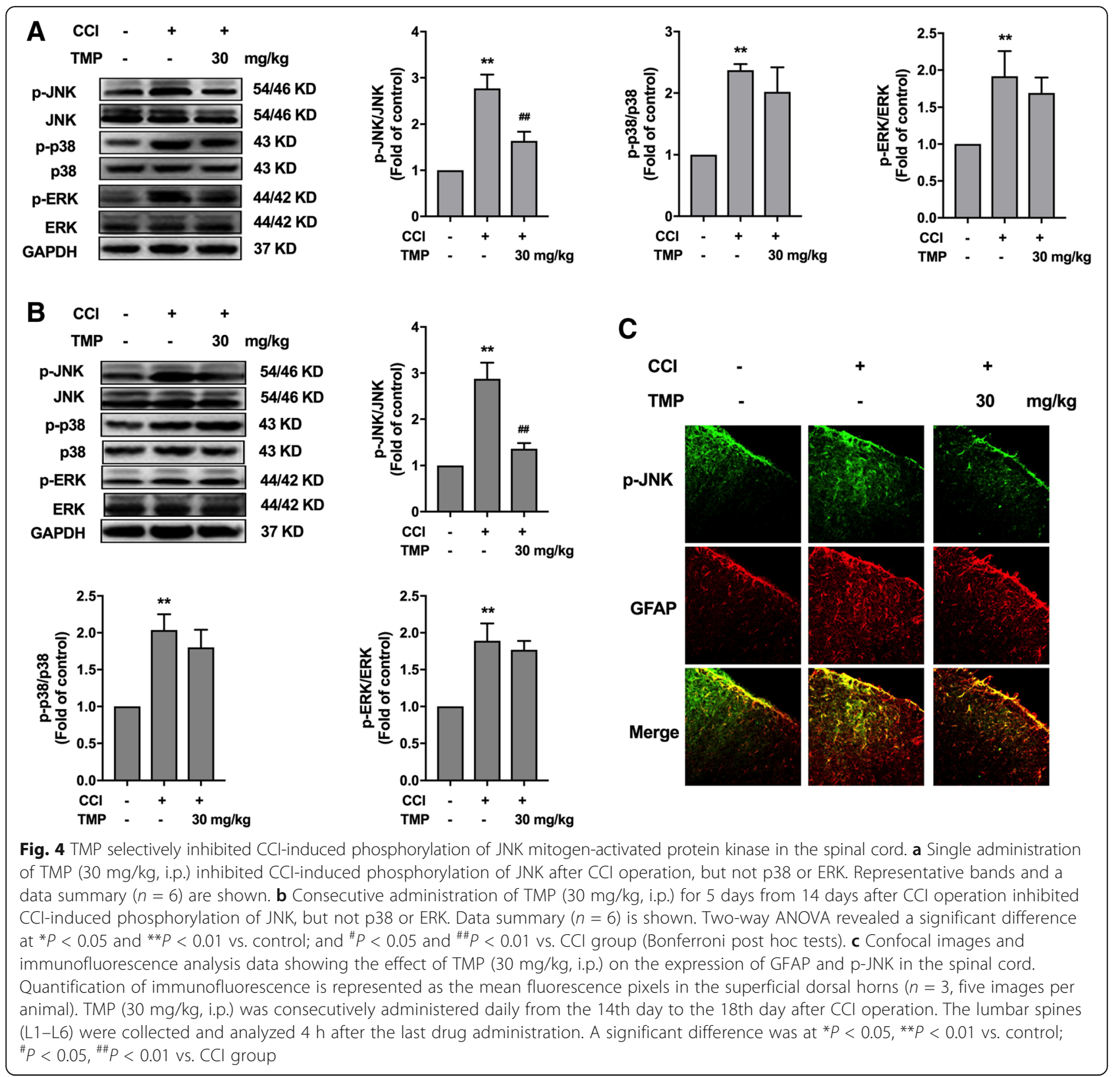

Furthermore, we found that JNK inhibitor and TAK1 inhibitor could suppress CCI-induced MMP-2 and MMP-9 (Fig. 6b). Administration of JNK and TAK1 inhibitor attenuated the maintenance CCI-induced neuropathic pain for the mechanical hyperalgesia thresholds, respectively (Fig. 6c).

TMP inhibits the allodynia induced by IL-1 $\beta$ in vivo The above results suggested that TMP, through TAK1/ JNK, may inhibit the astrocytes activation induced by proinfammatory factors. We further investigated the effects of TMP on the allodynia and astrocytes activation induced by IL-1 $\beta$. Injection of $10 \mu \mathrm{l}$ of IL-1 $\beta(20 \mathrm{ng} / \mathrm{ml}$, i.t.) into the spinal cord induced hyperalgia with a significant decrease in mechanical withdrawal. Treatment with TMP $(30 \mathrm{mg} / \mathrm{kg}$, i.p. $)$ at $30 \mathrm{~min}$ before IL-1 $\beta$ injection attenuated the hyperalgia. Similarly, the TAK1 inhibitor 5Z-7-oxozeaenol $(5 \mu \mathrm{g} / 10$ ul, i.t., at $30 \mathrm{~min}$ before IL-1 $\beta$ injection) and the JNK inhibitor SP600125 $(5 \mu \mathrm{g} / 10 \mathrm{ul}$, i.t., at $30 \mathrm{~min}$ before IL-1 $\beta$ injection) mimicked the ameliorative effects of TMP (Fig. 7a). Moreover, TMP, 5Z-7-oxozeaenol, and SP600125 suppressed the higher expression levels of GFAP after the injection of IL-1 $\beta$ (Fig. 7b). In addition, they could inhibit the increased MMP-2/9 and phosphorylation of JNK and TAK1 induced by IL-1 $\beta$ (Fig. 7c, d). 

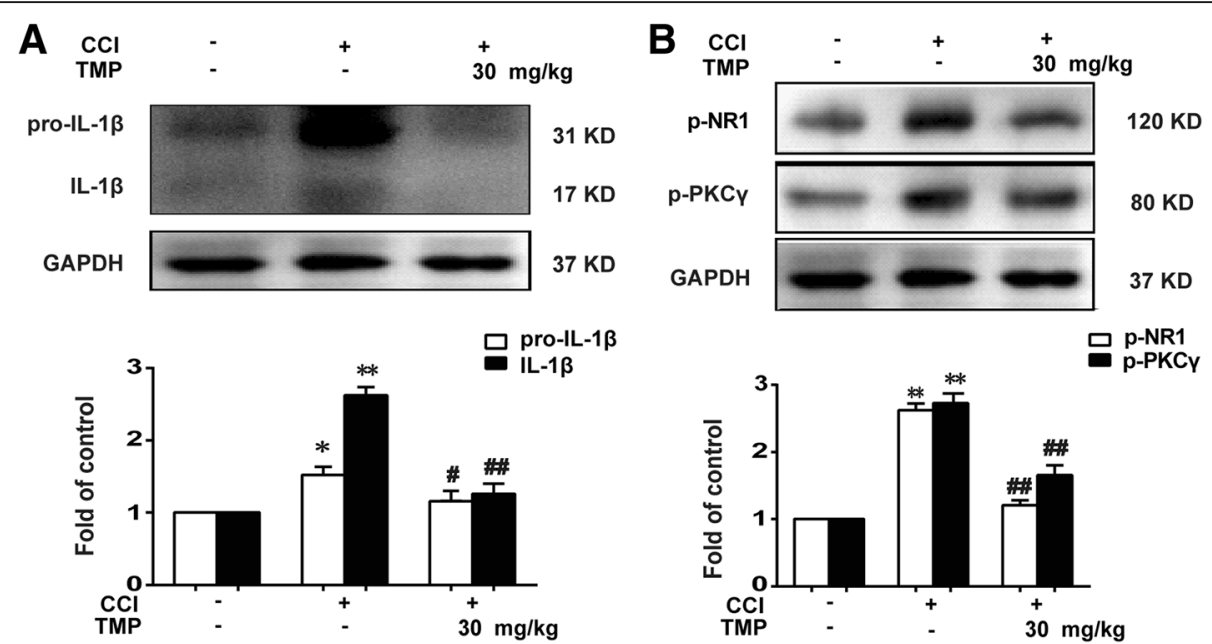

Fig. 5 TMP significantly attenuated CCl-induced neuron cell activation and IL-1 $\beta$ production in spinal cords. a Single administration of TMP significantly inhibited the cleavage of IL-1 $\beta$. TMP (30 mg/kg, i.p.) was administered on the 14th day after CCl operation. b Single administration of TMP inhibited CCl-induced phosphorylation of protein kinase C (PKC) y and N-methyl-D-aspartate receptor (NR) 1. TMP (30 mg/kg, i.p.) was administered on the 14th day after $\mathrm{CCl}$ operation. The western blot samples $(n=6)$ were collected at $4 \mathrm{~h}$ after TMP administration. A significant difference was at ${ }^{*} P<0.05$, ${ }^{*} P<0.01$ vs. control; ${ }^{*} P<0.05,{ }^{\# \#} P<0.01$ vs. CCl group

\section{A}

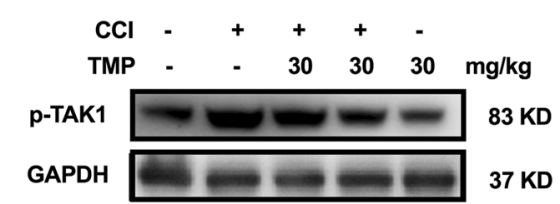

B

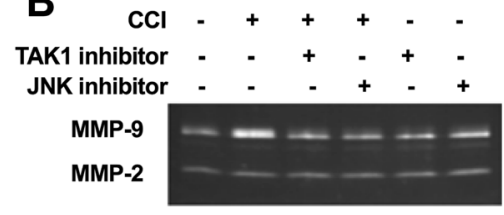

$92 \mathrm{KD}$ $72 \mathrm{KD}$

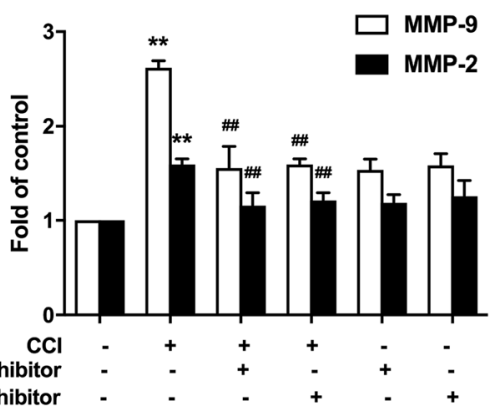

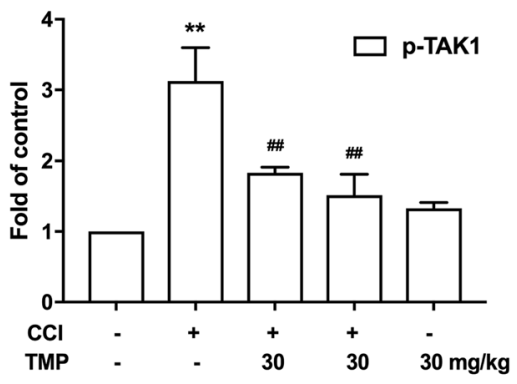

C
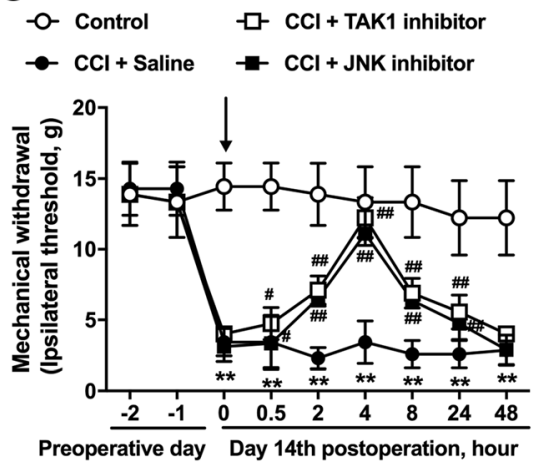

Fig. 6 TMP attenuated the level of pTAK1, which could be mimicked by TAK1 and JNK inhibitors. a Single administration of TMP (30 kg/mg, i.p) after CCl operation 14 days significantly suppressed CCl-induced TAK1 phosphorylation in the spinal cord. Consecutive administration of TMP (30 kg/mg, i.p) for 5 days from 14 days after CCI operation significantly attenuated CCl-induced TAK1 phosphorylation. The western blot samples $(n=6)$ were collected as described above. b Administration of TAK1 inhibitor $(5 \mu \mathrm{g} / 10 \mu \mathrm{l}$, i.t.) and JNK inhibitor $(5 \mu \mathrm{g} / 10 \mu \mathrm{l}$, i.t.) significantly inhibited CCl-induced MMP-2 and MMP-9. c Administration of JNK inhibitor and TAK1 inhibitor inhibited CCl-induced mechanical allodynia. A significant difference was at ${ }^{*} P<0.05,{ }^{* *} P<0.01$ vs. control; ${ }^{\#} P<0.05,{ }^{\# \#} P<0.01$ vs. CCl group 


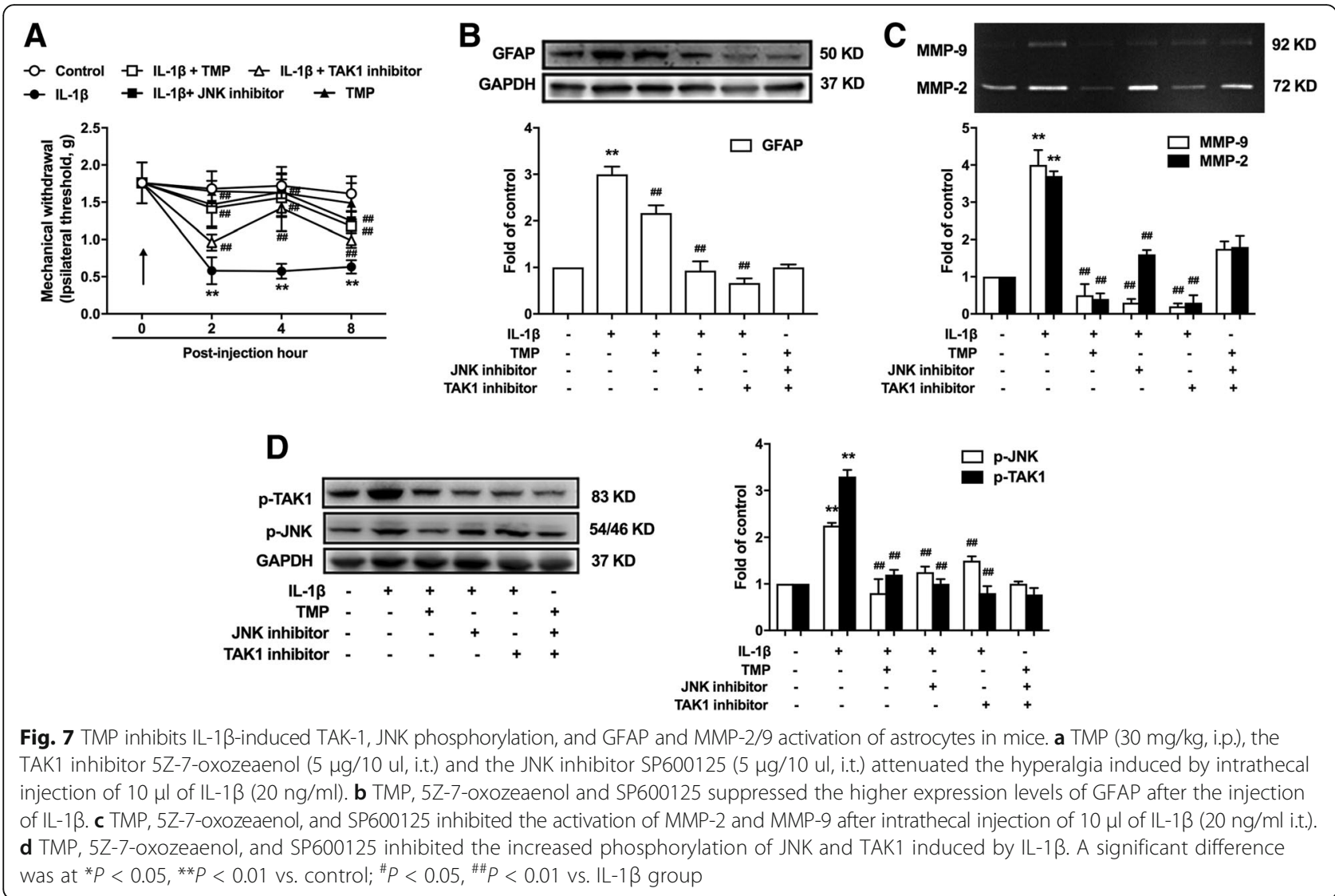

TMP inhibits IL-1 $\beta$-induced TAK-1 and JNK phosphorylation of astrocytes in vitro

To investigate the in vitro effects of TMP on proinflammatory factor-induced astrocyte activation, we used the immortalized murine astrocyte cell line C8, a clonal glial cell line derived from a rat glial tumor.

Before the treatment with IL-1 $\beta$ (20 ng/ml), C8 cells were pretreated with different doses $(0.5,5$, and $50 \mu \mathrm{M})$ of TMP for $30 \mathrm{~min}$. We then analyzed the C8 cells after IL-1 $\beta$ exposure. The group that was pre-administered with TMP $(0.5,5$, and $50 \mu \mathrm{M})$ significantly reduced TAK1 and JNK phosphorylation (Fig. 8). Based on the obtained results, the mechanisms of action and the underlying signaling pathways of TMP were proposed in Fig. 9.

\section{Discussion}

In this study, our major findings included the following: (1) CCI-induced increases in MMP-2/9 in astrocytes may be mediated by JNK, (2) TMP significantly attenuated the maintenance of CCI-induced mechanical allodynia, (3) TMP inhibited CCI-induced spinal astrocyte activation, (4) TMP significantly suppressed the expression of MMP-2/9, and (5) TMP selectively suppressed phosphorylation of JNK via TAK1 signaling pathway, but had no effects on ERK and p38.
Activated astrocytes and mediated neuroinflammation are critical targets for neuropathic pain [17]. Spinal microglial activation in both dorsal and ventral horns peak 1 week after injury followed by a slow decline over several weeks, and astrocytes play an important role in the maintenance of neuropathic pain [18]. Astrocyte reactions after nerve injury, arthritis, and tumor growth are more persistent than microglial reactions, and they display a better correlation with chronic pain behaviors [7]. Studies report that the inhibition of activation of astrocytes could effectively attenuate neuropathic pain [18]. Our data show that either a single dose or continuous administration of TMP could significantly and dosage dependently attenuate CCI-induced neuropathic pain (Fig. 1). We also demonstrated that TMP significantly inhibited the upregulation of GFAP expression (Fig. 2). These data are consistent with previous results showing that astrocyte reactions are associated with the maintenance of pain [18].

Previous studies have shown that MMP-9 and MMP-2 have been considered key molecules for the onset and maintenance of neuropathic pain inhibition of astrocytes activation and could effectively attenuate neuropathic pain [18]. It has also been shown that MMP-2/9 contributes to the cleavage of IL-1 $\beta$ [10]. Then, increased IL-1 $\beta$ facilitates $\mathrm{PKC} \gamma$ phosphorylation through IL-1 $\beta$ receptors, 

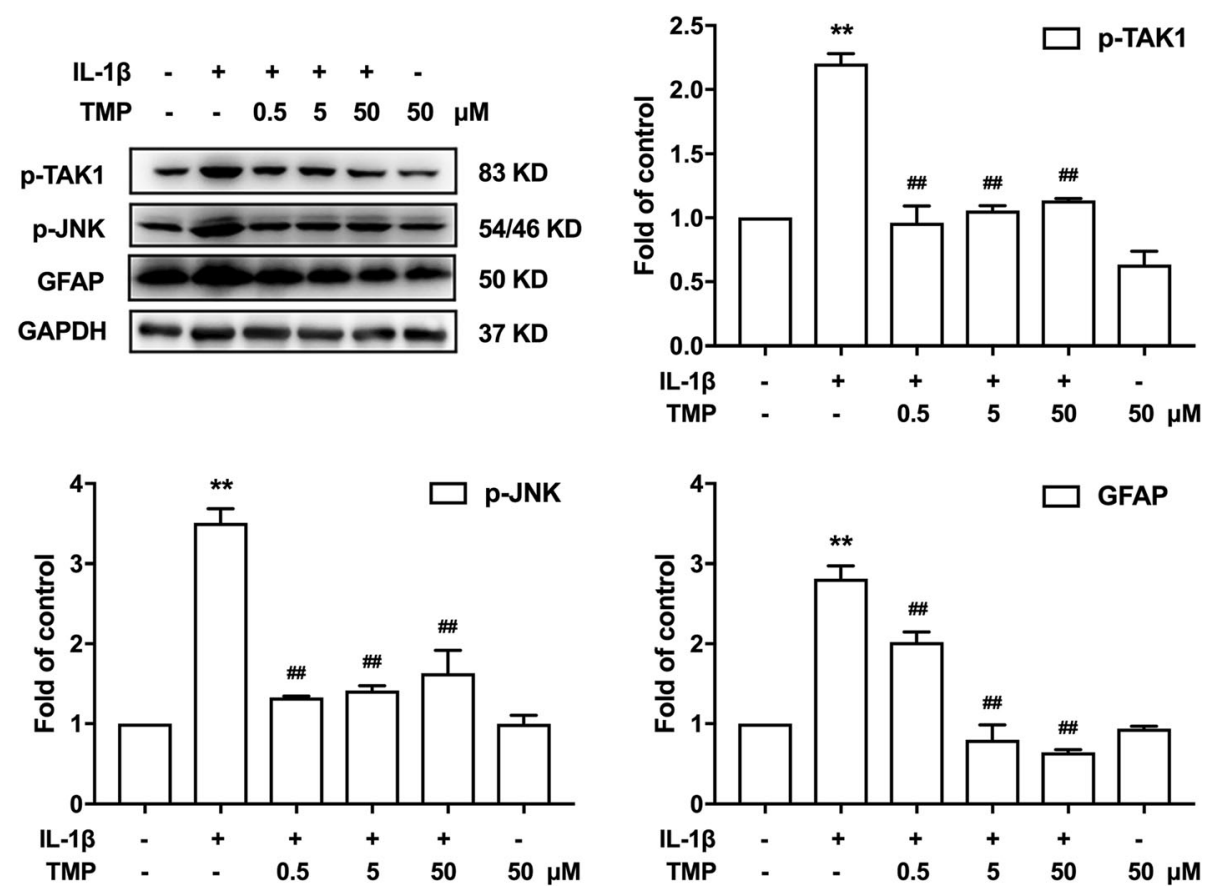

Fig. 8 TMP inhibits IL-1 $\beta$-induced TAK-1, JNK phosphorylation, and GFAP activation of astrocytes in vitro. Before the treatment of IL-1 3 (20 $\mathrm{ng} / \mathrm{ml})$, astrocyte cell line $\mathrm{C} 8$ were pretreated with different doses $(0.5,5$, and $50 \mu \mathrm{M})$ of TMP for $30 \mathrm{~min}$. TMP significantly reduced TAK-1, JNK phosphorylation, and GFAP. A significant difference was at ${ }^{*} P<0.05,{ }^{* *} P<0.01 \mathrm{vs}$. control; ${ }^{*} P<0.05,{ }^{\# \#} P<0.01 \mathrm{vs}$. IL-1 $\beta$ group

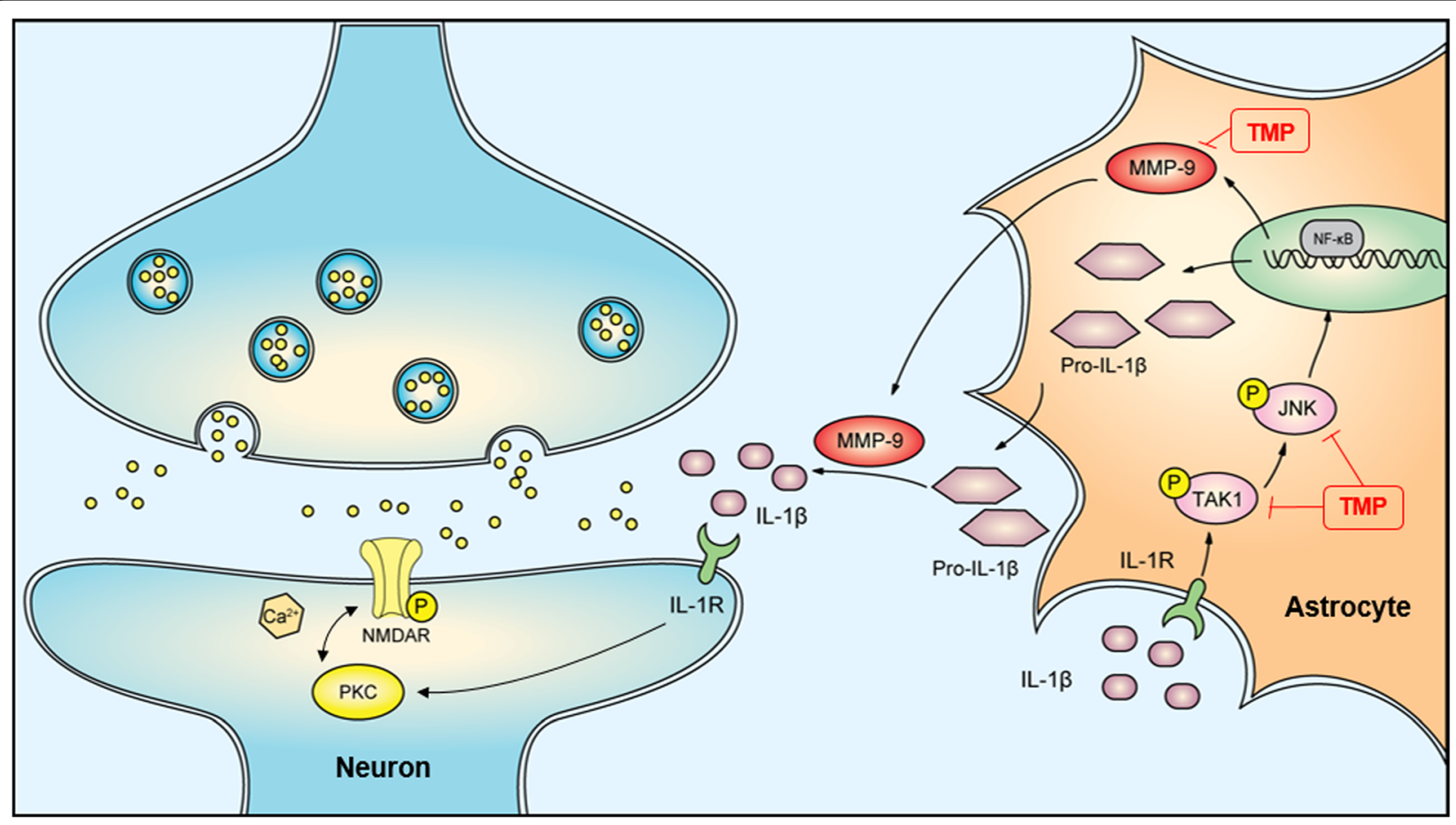

Fig. 9 Schematic indicating the suppression of JNK-MMP2/9 in astrocytes to alleviate neuropathic pain by TMP. After CCI surgery, several pathways including the release of IL-1 $\beta$, and calcium influx have been identified to signal the activation of astrocytes and neurons, leading to the neuroinflammation and central sensitization. TMP inhibited the MMP-2/9 activation, the maturation of IL-1 $\beta$, and the activation of PKC and NMDA. The inhibition of TAK1-JNK-MMP2/9 was a critical mechanism for analgesic effect of TMP in astrocytes 
leading to enhancement of NMDA receptor activity by NR1 subunit phosphorylation [19]. Activation of NMDA receptors subsequently induces $\mathrm{Ca}^{2+}$ influx and activates downstream signal cascade such as CaMKII [20]. These proteins can phosphorylate downstream molecules (e.g., $\mathrm{PKC} \gamma$ ), which in turn lead to further activation of the NMDA receptor and contribution to central sensitization [21]. Our results have shown that TMP treatment significantly reduced the activity of MMP-2/9 in vivo induced by CCI or IL-1 $\beta$ (Figs. 4 and 7). We have also shown that the TMP-inhibiting activity of MMP-2 is more intense than that of MMP-9 (Fig. 3). Moreover, our data indicate that CCI-induced increases of IL- $1 \beta$ in the spinal cord were significantly inhibited by TMP (Fig. 5a), which were reconciled with the data in Fig. 7. In addition, the phosphorylation level of NR1 and PKC could also be suppressed by TMP (Fig. 5b, c). Taken together, our results suggest that inhibition of MMP-2/9 and IL-1 $\beta$ and that following the NR1 and PKC signal cascades could attenuate neuropathic pain.

Our present study and previous reports show pivotal roles of MMP-2/9 in pain-producing molecular signals not only in neurons [10] but also in glial cells in chronic pain states [12]. We further explored the detailed mechanism of TMP in inhibiting MMP-2/9 in astrocytes. We focused on the mitogen-activated protein kinase (MAPK) families. MAPK families are important for regulating neural plasticity and inflammatory responses and play essential roles in chronic pain [22]. The MAPK family has three major members including extracellular signalregulated kinase (ERK), p38, and c-Jun N-terminal kinase (JNK) [23, 24]. Nerve injury or spinal cord injury induces a profound activation of MAPKs in the spinal cord [22]. Interestingly, MAPKs show a selective distribution by cell type. It is generally believed that activation of ERK occurs mainly in neurons, activation of p38 occurs mainly in microglia, and activation of c-Jun $\mathrm{N}$-terminal kinase (JNK) occurs mainly in astrocytes. Our data showed that TMP significantly inhibits activation of JNK in spinal cord astrocytes and has no effect on ERK and p38 phosphorylation according to the results of a western blot, which was reconciled with immunofluorescence results (Fig. 4). To further confirm the direct suppressive effects of TMP on JNK, we repeated the experiments in cultured astrocytes. TMP still inhibited IL- $1 \beta$-induced astrocyte activation, JNK phosphorylation, and the expression of MMP-2/9. Moreover, JNK inhibitor improved CCI-induced neuropathic pain (Fig. 6c) and inhibited MMP-2/9 levels (Fig. 6b).

We focused on the interactions between transforming growth factor-activated kinase 1 (TAK1) and TMP. We attended to the following aspects. First, TAK1 is a member of the MAPKKK family [25] and an upstream regulator of JNK [26]. Second, previous studies have already shown that TAK1 was mainly located in hyperactive astrocytes in the spinal cord after nerve injury [16]. Third, TAK1 was increased after nerve injury, and TAK1 inhibitor AS-ODN suppressed the activation of JNK in spinal astrocytes [16]. Our data indicates that after the CCI operation, the phosphorylation level of TAK1 increases and that TMP could suppress TAK1 expression in vivo (Fig. 6a). Further, administration of TAK1 inhibitor could attenuate neuropathic pain (Fig. 6c) and inhibit the expression of MMP-2/9 (Fig. 6b).

Our data suggest that TMP selectively suppressed the JNK signal pathway to inhibit the activation of astrocytes and then attenuated neuropathic pain via downregulation of TAK1 phosphorylation. However, it must be mentioned that we could not exclude downstream signaling pathway suppression, such as CaMKII. Previous studies have demonstrated that TMP could inhibit the activation of the calcium/calmodulin/calmodulin-dependent protein kinase $(\mathrm{Ca} 2+/ \mathrm{CaM} / \mathrm{CaMKII})$ pathway [27]. TMP may suppress CaMKII to attenuate neuropathic pain. The exact mechanisms involved with TMP and CaMKII require further study.

\section{Conclusions}

In summary, TMP could improve the maintenance of chronic constrictive injury (CCI)-induced neuropathic pain. Our results demonstrate that TMP regulates MMP-2 and MMP-9 signaling pathway in astrocytes by diminishing the phosphorylation of TAK1 and JNK, which are also important to improving neuropathic pain. Therefore, blocking the TAK1/JNK/MMPs signaling cascade in astrocytes might provide a fruitful strategy for treating intractable neuropathic pain. Altogether, our studies suggest that TMP may be a potential drug candidate for neuropathic pain treatment.

\section{Additional file}

Additional file 1: Figure S1. Additional controls with TMP treatment alone did not affect the baseline levels of p-p38, p-JNK, p-ERK MAPK family, IL-1 $\beta, p-N R 1$, and $p-P K C \gamma$ in vivo. Figure S2. TMP did not show a notable influence on the microglia marker IBA1. A significant difference was at ${ }^{*} P<0.05,{ }^{*} P<0.01$ vs. control. Figure S3. TMP did not show a notable influence on the MMP-2/9 in DRG. A significant difference was at ${ }^{*} P<0.05,{ }^{*} P<0.01$ vs. control. (DOCX $574 \mathrm{~kb}$ )

\section{Abbreviations}

BSA: Bovine serum albumin; DMEM: Dulbecco's modified Eagle's medium; DMSO: Dimethyl sulfoxide; ERK: Extracellular-regulated protein kinases; GAPDH: Glyceraldehyde-3-phosphate dehydrogenase; GFAP: Glial fbrillary acidic protein; IL-1ß: Interleukin-1 $\beta$; JNK: c-Jun N-terminal kinase; MAPK: Mitogen-activated protein kinase; MMP-2/9: Matrix metalloproteinase-2/9; NMDA: N-methyl-D-aspartic acid; NR1: N-methyl-Daspartate receptor 1; PBS: Phosphate-buffered saline; PKC: Protein kinase C; SDS-PAGE: Sodium dodecyl sulfate polyacrylamide gel electrophoresis; TAK1: Transforming growth factor beta-activated kinase 1; TMP: Tetramethylpyrazine 


\section{Acknowledgements}

Not applicable.

\section{Funding}

This work was supported by the National Natural Science Foundation of China (No. 81471142), National Science Foundation for Young Scientists of Jiangsu province (KY1010831161312010), and China Postdoctoral Science Foundation Commission (No. 2015M580473).

\section{Availability of data and materials}

The datasets during and/or analyzed during the current study are available from the corresponding author on reasonable request.

\section{Authors' contributions}

$\sqcup$ and $C P$ designed the experiments, performed the experiments, analyzed the results, and drafted the manuscript. BL carried out the behavioral measure. LL carried out the western blotting analysis. LH carried out the cell cultures. YH helped to draft the manuscript. WL conceived of the study, and participated in its design and coordination and helped to draft the manuscript. CW carried out the western blotting analysis, gelatin zymography, immunofluorescence, and RT-PCR when the article is revised. All authors read and approved the final manuscript.

\section{Ethics approval and consent to participate}

All procedures were strictly performed in accordance with the regulations of the ethics committee of the International Association for the Study of Pain and the Guide for the Care and Use of Laboratory Animals (The Ministry of Science and Technology of China, 2006). All animal experiments were approved by Nanjing Medical University Animal Care and Use Committee and were designed to minimize suffering and the number of animals used.

\section{Consent for publication}

Not applicable.

\section{Competing interests}

The authors declare that they have no competing interests.

\section{Publisher's Note}

Springer Nature remains neutral with regard to jurisdictional claims in published maps and institutional affiliations.

\section{Author details}

'Department of Pharmacology, Jiangsu Key Laboratory of Neurodegeneration, Nanjing Medical University, 140 Han-Zhong Road, Nanjing 210029, China. 'Department of Ophthalmology, the First Affiliated Hospital with Nanjing Medical University, Nanjing 210029, China. ${ }^{3}$ Jiangsu Province Key Laboratory of Anesthesiology, Xuzhou Medical College, Xuzhou 221000, China. ${ }^{4}$ Department of pain, Shandong Oianfoshan Hospital, Shandong 250014, China. ${ }^{5}$ Department of Gynecologic Oncology, Jiangsu Institute of Cancer Research, Jiangsu Cancer Hospital, Nanjing Medical University Affiliated Cancer Hospital, Nanjing, Jiangsu 210009, China.

Received: 24 January 2017 Accepted: 23 August 2017

Published online: 31 August 2017

\section{References}

1. Tan T, Barry PW, Reken S, Baker M. Pharmacological management of neuropathic pain in non-specialist settings: summary of NICE guidance. BMJ. 2010:340:707-9.

2. Egeblad M, Werb Z. New functions for the matrix metalloproteinases in cancer progression. Nat Rev Cancer. 2002;2:161-74.

3. David $\mathrm{S}$, Kroner A. Repertoire of microglial and macrophage responses after spinal cord injury. Nat Rev Neurosci. 2011;12:388-99.

4. Zhang J, De Koninck Y. Spatial and temporal relationship between monocyte chemoattractant protein-1 expression and spinal glial activation following peripheral nerve injury. J Neurochem. 2006;97:772-83.

5. Ledeboer A, Sloane EM, Milligan ED, Frank MG, Mahony JH, Maier SF, Watkins LR. Minocycline attenuates mechanical allodynia and proinflammatory cytokine expression in rat models of pain facilitation. Pain. 2005;115:71-83.

6. Milligan ED, Watkins LR. Pathological and protective roles of glia in chronic pain. Nat Rev Neurosci. 2009;10:23-36.
7. Ji RR, Berta T, Nedergaard M. Glia and pain: is chronic pain a gliopathy? Pain 2013;154(Suppl 1):S10-28.

8. Roomi MW, Monterrey J, Kalinovsky T, Rath M, Niedzwiecki A. Distinct patterns of matrix metalloproteinase-2 and -9 expression in normal human cell lines. Oncol Rep. 2009;21:821-6.

9. Vallejo R, Tilley DM, Vogel LA, Benyamin RM. The role of glia and the immune system in the development and maintenance of neuropathic pain. Pain Practice. 2010;10:167-84.

10. Kawasaki Y, Xu Z, Wang X, Park JY, Zhuang Z, Tan P, Gao Y, Roy K, Corfas G, Lo EH. Distinct roles of matrix metalloproteases in the early-and late-phase development of neuropathic pain. Nat Med. 2008;14:331-6.

11. Ji R, Xu Z, Wang $X$, Lo EH. Matrix metalloprotease regulation of neuropathic pain. Trends Pharmacol Sci. 2009:30:336-40

12. Liu W, Han Y, Liu Y, Song AA, Barnes BE, Song X. Spinal matrix metalloproteinase-9 contributes to physical dependence on morphine in mice. J Neurosci. 2010;30:7613-23.

13. Rooprai HK, Rucklidge GJ, Panou C, Pilkington GJ. The effects of exogenous growth factors on matrix metalloproteinase secretion by human brain tumour cells. Br J Cancer. 2000;82:52-5.

14. Chen Z, Pan X, Georgakilas AG, Chen P, Hu H, Yang Y, Tian S, Xia LP, Zhang J, Cai X. Tetramethylpyrazine (TMP) protects cerebral neurocytes and inhibits glioma by down regulating chemokine receptor CXCR4 expression. Cancer Lett. 2013;336:281-9.

15. Vu TH, Shipley JM, Bergers G, Berger JE, Helms JA, Hanahan D, Shapiro SD, Senior RM, Werb Z. MMP-9/gelatinase B is a key regulator of growth plate angiogenesis and apoptosis of hypertrophic chondrocytes. Cell. 1998;93:411-22.

16. Katsura H, Obata K, Miyoshi K, Kondo T, Yamanaka H, Kobayashi K, Dai Y, Fukuoka T, Sakagami M, Noguchi K. Transforming growth factor-activated kinase 1 induced in spinal astrocytes contributes to mechanical hypersensitivity after nerve injury. Glia. 2008;56:723-33.

17. Gao Y, Ji R. Targeting astrocyte signaling for chronic pain. Neurotherapeutics, 2010;: :482-93.

18. Scholz J, Woolf CJ. The neuropathic pain triad: neurons, immune cells and glia. Nat Neurosci. 2007;10:1361-8.

19. Malmberg AB, Chen C, Tonegawa S, Basbaum Al. Preserved acute pain and reduced neuropathic pain in mice lacking PKCy. Science. 1997;278:279-83.

20. Zhuo M, Wu G, Wu L. Neuronal and microglial mechanisms of neuropathic pain. Molecular Brain. 2011:4:31.

21. Hucho T, Levine JD. Signaling pathways in sensitization: toward a nociceptor cell biology. Neuron. 2007;55:365-76.

22. Ji R, Gereau RW, Malcangio M, Strichartz GR. MAP kinase and pain. Brain Res Rev. 2009;60:135-48.

23. Liu Y, Shepherd E, Nelin LD. MAPK phosphatases—regulating the immune response. Nat Rev Immunol. 2007;7:202-12.

24. Zhuang ZY. A peptide c-Jun N-terminal kinase (JNK) inhibitor blocks mechanical allodynia after spinal nerve ligation: respective roles of JNK activation in primary sensory neurons and spinal astrocytes for neuropathic pain development and maintenance. J Neurosci. 2006;26:3551-60.

25. Ajibade AA, Wang HY, Wang R. Cell type-specific function of TAK1 in innate immune signaling. Trends Immunol. 2013;34:307-16.

26. Adhikari A, Xu M, Chen ZJ. Ubiquitin-mediated activation of TAK1 and IKK Oncogene. 2007;26:3214-26.

27. Zhao H, Xu M, Zhang Q, Guo Z, Peng Y, Qu Z, Li Y. Tetramethylpyrazine alleviated cytokine synthesis and dopamine deficit and improved motor dysfunction in the mice model of Parkinson's disease. Neurol Sci. 2014;35:1963-7. 